\title{
APPLICATION OF THERMAL CONDUCTION MODELS TO DEEPSEA DISPOSAL OF RADIOACTIVE WASTES
}

W, P, SCHIMMEL, JR, AND C, E, HICKOX

Prepared by Sandia Laboratories, Albuquerque. New Mexico 87115

and Livermore, California 94550 for the United States Energy Research

and Development Administration under Contract AT (29-1)-789

Printed March 1978

\section{Sandia Laboratories}




\section{DISCLAIMER}

This report was prepared as an account of work sponsored by an agency of the United States Government. Neither the United States Government nor any agency Thereof, nor any of their employees, makes any warranty, express or implied, or assumes any legal liability or responsibility for the accuracy, completeness, or usefulness of any information, apparatus, product, or process disclosed, or represents that its use would not infringe privately owned rights. Reference herein to any specific commercial product, process, or service by trade name, trademark, manufacturer, or otherwise does not necessarily constitute or imply its endorsement, recommendation, or favoring by the United States Government or any agency thereof. The views and opinions of authors expressed herein do not necessarily state or reflect those of the United States Government or any agency thereof. 


\section{DISCLAIMER}

Portions of this document may be illegible in electronic image products. Images are produced from the best available original document. 
Issued by Sandia Laboratories, operated for the United States Department of Energy by Sandia Corporation.

\section{NOTICE}

This report was prepared as an account of work sponsored by the United States Government. Neither the United States nor the Department of Energy, nor any of their employees, nor any of their contractors, subcontractors, or their employees, makes any warranty, express or implied, or assumes any legal liability or responsibility for the accuracy, completeness or usefulness of any information, apparatus, product or process disclosed, or represents that its use would not infringe privately owned rights. 
SAND77-0.752

Unlimited Release

Printed March 1978

APPLICATION OF THERMAL CONDUCTION MODELS

TO DEEPSEA DISPOSAL OF RADIOACTIVE WASTES

\author{
Walter P. Schimmel, Jr., and Charles E. Hickox \\ Fluid and Thermal Sciences Department 1260 \\ Sandia Laboratories \\ Albuquerque, NM 87115
}

\begin{abstract}
Thermal problems associated with the emplacement of radioactive wastes in the deepsea sedimentary layer have been studied. In particular, the nature of the temperature field surrounding and the interstitial water velocity arising from a buried cask have been examined. Worst case estimates indicate that the velocity will be extremely weak and thus not likely to provide a primary transport mechanism for the radioactive material. This statement will, of course, only apply for moderately low levels of heat generation by the decaying radio nuclides. Because of the low interstitial water velocity, thermal conduction models can be used to predict the temperature field in the surrounding sediments as well as the cask surface temperature. This is equivalent to "decoupling" the energy and momentum conservation relationships thus simplifying the solution of the temperature field.

The present work considers in some detail the temperature field surrounding a vertical circular "cylinder" located a distance below a horizontal, isothermal, plane surface. Actually, the isotherm corresponding to the cask surface is an ellipsoid of revolution but the error will be small for large values of the length to diameter ratio. The resulting expression can be used to estimate temperature of the cask surface for material degradation studies and the effect of temperature upon the ion transport process in the sediments.
\end{abstract}


TABLE OF CONTENTS

Page

INTRODUCTION 2

THERMAL CONDUCTION MODELS 6

LABORATORY RESULTS 13

CONCLUSIONS 24

REFERENCES

APPENDIX 
INTRODUCTION

The "energy crisis" of the 1970's has made nearly every American citizen aware of the finite extent of our fossil fuel reserves. Because of this, ever increasing dependence must be placed upon alternatives for power generation. of the near-term alternatives, virtually everyone agrees that light water fission reactors (LWR) represent the replacement source with the highest probability of success.

Although the potentially attractive schemes of utilizing solar, geothermal and tidal energy are presently being investigated, it is unlikely that any of these will have a significant impact upon the energy shortage for the near-term future. Likewise, the technological problems which exist in the field of controlled thermonuclear fusion make the prospect of contemporary power generation by this process rather dim. Nuclear reactors, on the other hand, exist and are generating electrical power today.

Unfortunately (or fortunately depending on your vantage point), the need for widespread dissemination of the LWR's comes on the heels of discovery that the environment is also finite in terms of its capability to handle pollutants. The power engineer must now be aware of "cost" in the broader sense of the word. Not only cost as associated with the capital expenditures of acquiring, transporting and processing a fuel but the ultimate strain on the environment because of using the fuel. 
Nuclear fission reactors have their own form of costs in terms of ecological impact. One of these costs is the production of:highlevel radioactive waste (HLW) products. These waste products require almost perfect segregation from the environment. ${ }^{1}$. Because some of the constituents are extremely long-lived such as ${ }^{239} \mathrm{Pu}$ ' with a half-life of 24,000 years and ${ }^{129}$ I with a half-life of about 10 million years, they must be contained virtually forever. There is presently no evidence that any known material will be able to withstand the combined thermal and radioactive levels for more than a small fraction of the lifetimes of the toxic materials. It is therefore necessary to place as many barriers as possible between the reactor wastes and mankind's environment. The technology for transmutation of the long-lived isotopes or disposing of them outside the earth's atmosphere appears to be out of reach for the near term and thus will not be considered.

of the terrestrial sites considered for possible use as repositories, the sea floor appears particularly attractive. There exist regions in the world's oceans of low biomass and relative geological stability. In addition, the desirable features of fairly deepiwater $(4-6 \mathrm{~km})$ and low non-tidal currents near the bottom $(<5 \mathrm{~cm} / \mathrm{s})$ suggest the use of the mid-plate/gyre (MPG) regions. This view has been previously presented in Referencic 1 and 2. Uther areas in the sea could also be considered but the criteria listed above have eliminated most of them.

Initially, the thinking on seabed disposal was that the HEW would be simply "dumped" on the ocean bottom. ${ }^{3}$ Because of the; remoteness of the dump site, any toxic wastes which escaped the cask 
and waste form would be diluted before reaching mankind. This thinking dominates the studies of physical oceanographers for the past few years. ${ }^{4-6}$ Based on a recent investigation into the kinetics of thermally induced buoyant plumes, ${ }^{7}$ it appears that neither the dilution mechanism nor the specific release pathway to man is well known. One is, therefore, led to the next available option in seabed disposal--the emplacement of the HLW in the clay rich sediments underlying the MPG regions. The sediment can provide a "barrier" in terms of ion transport in a properly engineered disposal program. The present study will consider the thermal effects arising from the emplacement of a localized heat source in the deep ocean sediments. Of particular interest to the waste disposal program are the temperature of the waste form cask and the velocity of the interstitial water in the sediments. Cask temperature and that of the surrounding medium will dictate the rates at which the various chemical reactions occur. Sorption coefficients in the sediments will also be modified by the presence of the temperature field. Interstitial water motion is a key parameter in the leach rate studies which determine the effectiveness of the sediment column as a barrier.

In the sections to follow, a series of theoretical attempts to characterize the situation will be presented. Because of the extremely low permeabilities in the largely clay sediments, it is expected that the temperature field will be essentially that of a conduction-dominated situation. This, as opposed to a convectiondominated situation in which the temperature field would exhibit a strong "plume-like" behavior. It is useful, therefore, to develop 
thermal conduction models which ignore the velocity field and its resulting effect upon the temperature field. One particular geometric configuration, namely, a vertical finite length cylinder located some distance below a horizontal isothermal plane will be discussed in detail. This model obviously corresponds to the actual emplacement configuration. Results from the porous media convection studies ${ }^{8,9}$ confirm the conduction-like temperature field for the idealized source configurations considered.

\section{THERMAL CONDUCTION MODELS}

The actual configuration likely to be encountered in the deepsea disposal program is that of a finite length cylinder located some distance below the horizontal plane of the seabed. Present candidates for the emplacement process dictate that the axis of the. cylinder will probably be vertical. If permitted to sink under its own weight, however, the cask could be horizontal. Both these cases, in addition to some more "idealized" configurations (in the mathematical sense), will be presented. of the models considered, only the mathematical idealization of an infinite length cylinder will be used to predict transient behavior of the temperature field. This lack of transient information is not a serious consideration if the stable equilibrium of the emplaced cask is certain. If, however, some time-dependent stability mechanism takes place, it may be desirable to consider a transient description of the problem. An excellent compilation of transient conduction solutions which could be applied to the problem has recently been published. ${ }^{10}$ These, 
solutions are somewhat more tedious than the present time independent ones but they could be used if required.

An additional point that should be made is that constant uniform heat flux is assumed over the cannister lateral surface. If the actual decaying source behavior is assumed, these models could be used in a piecewise fashion ${ }^{11}$ to determine temperature response. Because it is likely that an emplacement engineer would use more sophisticated analytical (numerical) models for designing the cask and waste form, the present models represent only first-cut approximation to the actual situation.

The cylindrical conduction models to be presented are: (1) an infinite length cylinder in an infinite medium, (2) an infinite length cylinder in a finite medium, (3) a finite length cylinder in an infinite medium, and (4) a finite length vertical axis cylinder located some distance below an isothermal plane surface.

The first expression will be useful for short times but is has no equilibrium (infinite time) solution. The other three cases have only equilibrium solutions. The fourth solution is most applicable to the question of seabed disposal.

Assuming constant properties, and a constant heat flux at radius $R$, the solution for an infinite length cylinder in an infinite medium is given in Reference 12:

$T_{s}-T_{0}=\frac{-2 q}{\pi k} \int_{0}^{\infty}\left(1-e^{-\alpha x^{2} t}\right)\left\{\frac{J_{0}(x r) Y_{0}(x R)-Y_{0}(x r) J_{0}(x R)}{x^{2}\left[J_{I}^{2}(x R)+Y_{I}^{2}(x R)\right]}\right\} d x$ 
The solution for small values of $\xi=\frac{\alpha t}{R^{2}}$ evaluated at $r=R$ is,

$$
\mathrm{T}_{\mathrm{s}}-\mathrm{T}_{0}=\frac{\mathrm{Rq}}{\mathrm{k}}\left\{2 \xi^{\frac{1}{2}}\left[\operatorname{ierfc}(0)-\xi^{\frac{1}{2}} \mathrm{i}^{2} \operatorname{erfc}(0)+\ldots\right]\right\} \ldots
$$

Inserting the tabilated values of the complimentary error function integrals evaluated at zero yields,

$$
\mathrm{T}_{\mathrm{s}}-\mathrm{T}_{\mathrm{o}}=\frac{\mathrm{Rq}}{\mathrm{k}}\left\{2 \xi^{\frac{1}{2}}\left[.5642-\xi^{\frac{1}{2}}(.25)+\ldots\right]\right\}
$$

Likewise for large values of $\xi$,

$$
T_{S}-T_{0}=\frac{R q}{k}\left\{\frac{1}{2} \ln \left(\frac{4 \xi}{C}\right)+\frac{1}{4 \xi} \ln \left(\frac{4 \xi}{C}\right)+\ldots\right\} \text {. }
$$

where,

$$
c=e^{\gamma}=1.7811 \ldots .
$$

and $\gamma$ is Euler's constant; $0.57722 \ldots$.

This expression for long times will be referred to later because it is the same result one obtains when considering an infinite length Iine source emitting $(\mathrm{k} / \alpha) \mathrm{Q}$ units of heat per unit time per unit length. This result is usually written, 12

$$
T_{g}-T_{0}=-\frac{Q}{4 \pi \alpha} \operatorname{Ei}\left(\frac{-r^{2}}{4 \alpha t}\right) \text {, }
$$

where,

$$
E_{i}(x)=\int_{-\infty}^{x} e^{u} \frac{d u}{u}
$$

is the exponential integral and, 


$$
-E_{i}(x)=\gamma^{\prime}+\ln (x)-x+\frac{x^{2}}{4}+\ldots .
$$

For large values of the argument, this becomes;

$$
T_{S}-T_{0}=\frac{Q}{4 \pi \alpha}\left[\ln \left(\frac{4 \alpha t}{R^{2}}\right)-\ln c\right] \text {, }
$$

where $\operatorname{lnC}=\gamma$.

Substituting that,

$$
\frac{\mathrm{k}}{\alpha} \mathrm{Q}=2 \pi \mathrm{Rq}
$$

results in

$$
\mathrm{T}_{\mathrm{S}}-\mathrm{T}_{\circ}=\frac{\mathrm{gR}}{2 \mathrm{k}} \ln \left(\frac{4 \alpha \mathrm{t}}{\mathrm{CR}}\right)
$$

which is the same as Equation (4) for at least the first term. Note that the equilibrium or infinite time solution for the cylinder increases without bound and thus there is a point beyond which the expression fails to describe an actual physical case.

The methods of potential theory and the technique of images can be used to deduce an equilibrium solution for an infinite length cylinder located a distance below a surface parallel to its axis. This is given in Reference 13.

$$
\mathrm{T}_{\mathrm{S}}-\mathrm{T}_{\mathrm{O}}=\frac{\mathrm{qR}}{\mathrm{k}} \ln \left[\mathrm{h}+\sqrt{\mathrm{h}^{2}-1}\right] \text {, }
$$

where $h=H / R$ which for $H \ddot{R}$ becomes, 


$$
T_{S}-T_{0} \simeq \frac{q R}{k} \ln (2 h)
$$

This result is clearly finite indicating that the presence of a surface has a stabilizing effect upon the cylinder surface temperature.

The third case to be considered in this section is that of a finite cylinder in an infinite medium: Reference 14 presents the solution for this case,

$$
T_{S}-T_{0}=\frac{q R}{k} \ln \left(\ell+\sqrt{\ell^{2}+1}\right) \text {, }
$$

where $\ell=L / D$, the ratio of length to diameter of the cylinder. Again, this solution will be finite indicating that limiting the length of the cylinder also stabilizes its surface temperature. The final case to be considered is a vertical axis cylinder of length $L$ and diameter $D$ lncated a dictance $H$ below a horizontal isothermal plane surface. Details of the analytical procedure are presented in the appendix. In essence, the analysis follows the procedure outlined in Reference 15 for zero burial depth, and in fact, corrects some errors in the reference. The temperature of the cylinder predicted by this model is,

$$
\frac{T}{Q^{\prime} / 2 \pi k}=\ln \left(\ell+\sqrt{\ell^{2}-1}\right)+\frac{1}{2} \ln \left[\frac{2 h+3 n-r_{3}}{2 h+n-r_{4}}\right] \text {. }
$$

where the notation is the same as that of the previous models, except, 


$$
\begin{gathered}
\ell=\mathrm{H} / \mathrm{b}, \\
\mathrm{n}=\sqrt{\ell^{2}-1}, \\
r_{3}=\sqrt{1+(2 \mathrm{~h}+3 \mathrm{n})^{2}}, \\
\mathrm{~b}=\mathrm{D}\left(\frac{\ell+\frac{1}{2}}{\frac{2+2 \ell \sin ^{-1} \varepsilon}{\varepsilon}}\right)^{\frac{2}{2}},
\end{gathered}
$$

and,

$$
\varepsilon=\frac{1}{\ell} \sqrt{\ell^{2}-1}
$$

Some results of this expression are presented in Figure: 1 for various $\ell$ and $h$. Note that as $h$ tends to infinity, Equation (14) reduces to Equation (13) except for the sign beneath the square root. It is not clear why this descrepancy exists but its effect is vanishingly small as $\ell$ becomes large. The smallest $\ell$ considered in the present work is two.

The experimental results to be presented in a later section of. this report involve a spherical heater. For the case of pure conduction, it is possible to obtain a closed form analytical expression for the temperature profile throughout the conducting medium. For constant thermal properties and a constant heat flux at radius, $R$, the solution is given in Reference 12 , 


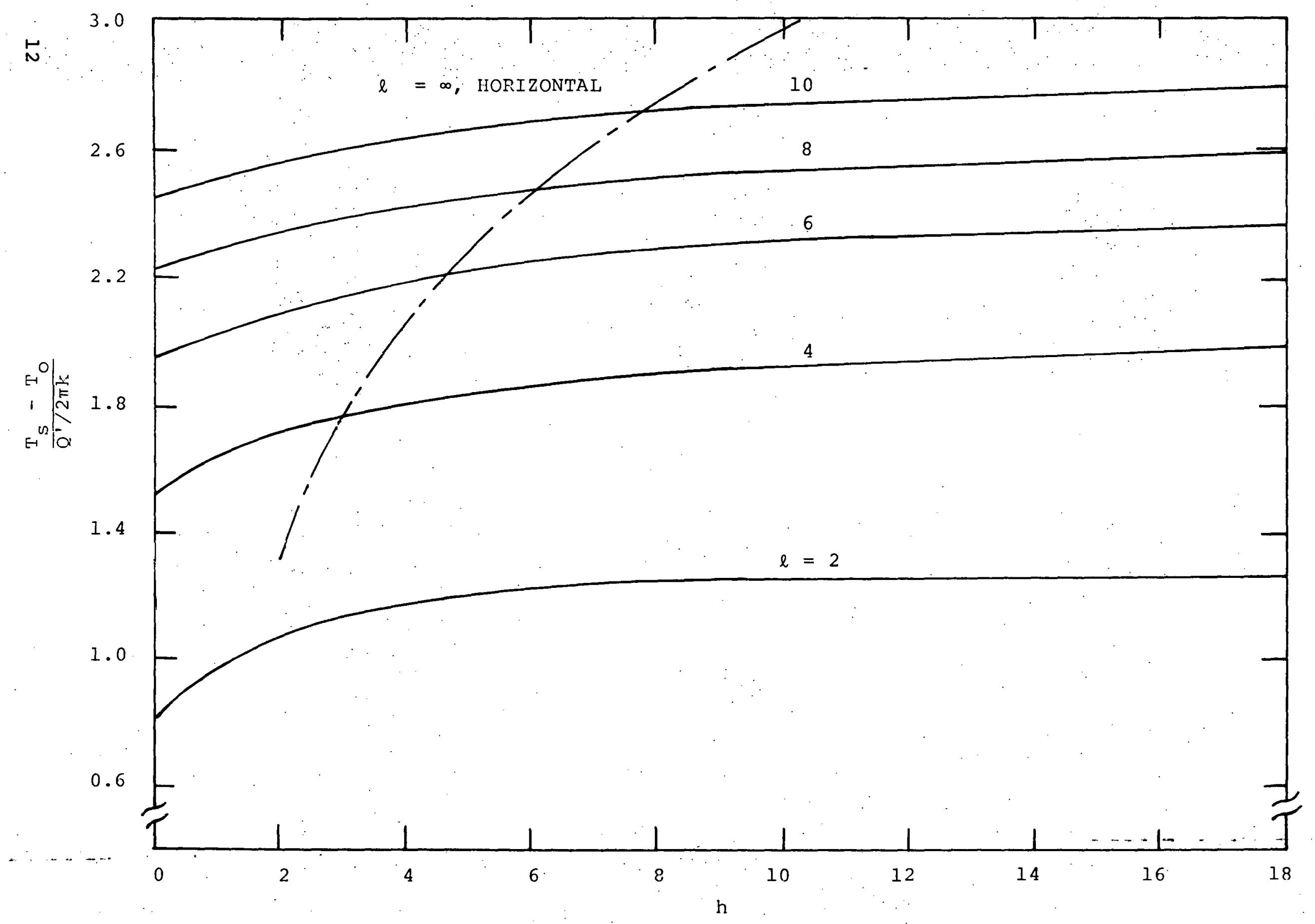

FIGURE 1. EFFECT OF BURIAL DEPTH AND ASPECT RATIO 


$$
T_{S}-T_{0}=\frac{R q}{k}\left\{\operatorname{erfc}\left(\frac{r-R}{2 \sqrt{\alpha t}}\right)-e^{\left(\frac{r-R}{R}+\frac{\alpha t}{R^{2}}\right)} \operatorname{erfc}\left[\frac{r-R}{2 \sqrt{\alpha t}}+\frac{\sqrt{\alpha t}}{R}\right]\right\}
$$

which when evaluated at $R$ becomes,

$$
\mathrm{T}_{s}-\mathrm{T}_{0}=\frac{\mathrm{Rg}}{\mathrm{k}}\left(1-\mathrm{e}^{\xi} \operatorname{erfc} \sqrt{\xi}\right) \quad
$$

For large values of $\xi=\frac{\alpha t}{R^{2}}$, the solution approaches

$$
T_{S}-T_{O}+\frac{R g}{k}
$$

If, now: a surface is considered in the medium and the sphere is located $\mathrm{H}$ below it, the expression is given in Reference 14,

$$
\mathrm{T}_{\mathrm{s}}-\mathrm{T}_{\mathrm{O}}=\frac{\mathrm{Rq}}{\mathrm{k}}\left(1-\frac{\mathrm{R}}{4 \mathrm{H}}\right) \text {. }
$$

Note that the presence of the surface modifies the equilibrium temperature of the sphere by a small amount for $H \gg R$. In fact, if $H=100 \mathrm{R}$ for example, the results for the transient case of the sphere in the infinite medium,will be within $1 \%$ of the actual case.

\section{LABORATORY RESULTS}

A spherical heater, $3.18 \mathrm{~cm}$ in diameter, was placed in a box of deep ocean sediments and its temperature-time history recorded. The dimensions of the plexiglass box are $30.5 \mathrm{~cm} \times 30.5 \mathrm{~cm} \times 35.6 \mathrm{~cm}$ deep and the sphere was placed 26.7. cm below the surface of the sediments. Initially, the heater flux was $0.62 \mathrm{w} / \mathrm{cm}^{2}$ but it was later lowered 
to be certain that the boiling temperature of the water was not reached. Figure 2 is the early portion of the temperature response curve. To help visualize the convective motion, a small gelatin, capsule of waterproof ink was placed vertically above the sphere with the surfaces of the sphere and the capsule touching. This technique was used successfully in the saturated coarse sand experiments to be described later. The utility of introducing the dye this way is that no momentum component due to injection is present indlcating that any transport is due to natural convection. Waterpronf ink was used because the sediments had a remarkable ability to completely absorb the color of all the food dyes and other materials which were initially used. Thermocouples were placed at several locations within the mud but the main region of interest was at the surface of the sphere.

The experiment was run continuously for 72 hours. In the final equilibrium situation, the heater was set at about 8 watts 10.25 : $w / \mathrm{cm}^{2}$ ). The temperature rise of the sphere over that of the sediment layer adjacent to the box wall in the horizontal direction was $4.7 .2 \mathrm{~K}$. The so-called electrical analogy for heat transfer states that,

$$
Q=\frac{\Delta T}{R_{t}}=q A_{s}
$$

where $R_{t}$ is a thermal resistance. For the case of pure conduction, the resistance is,

$$
R_{t}=\frac{L}{k A_{s}} \text {, }
$$




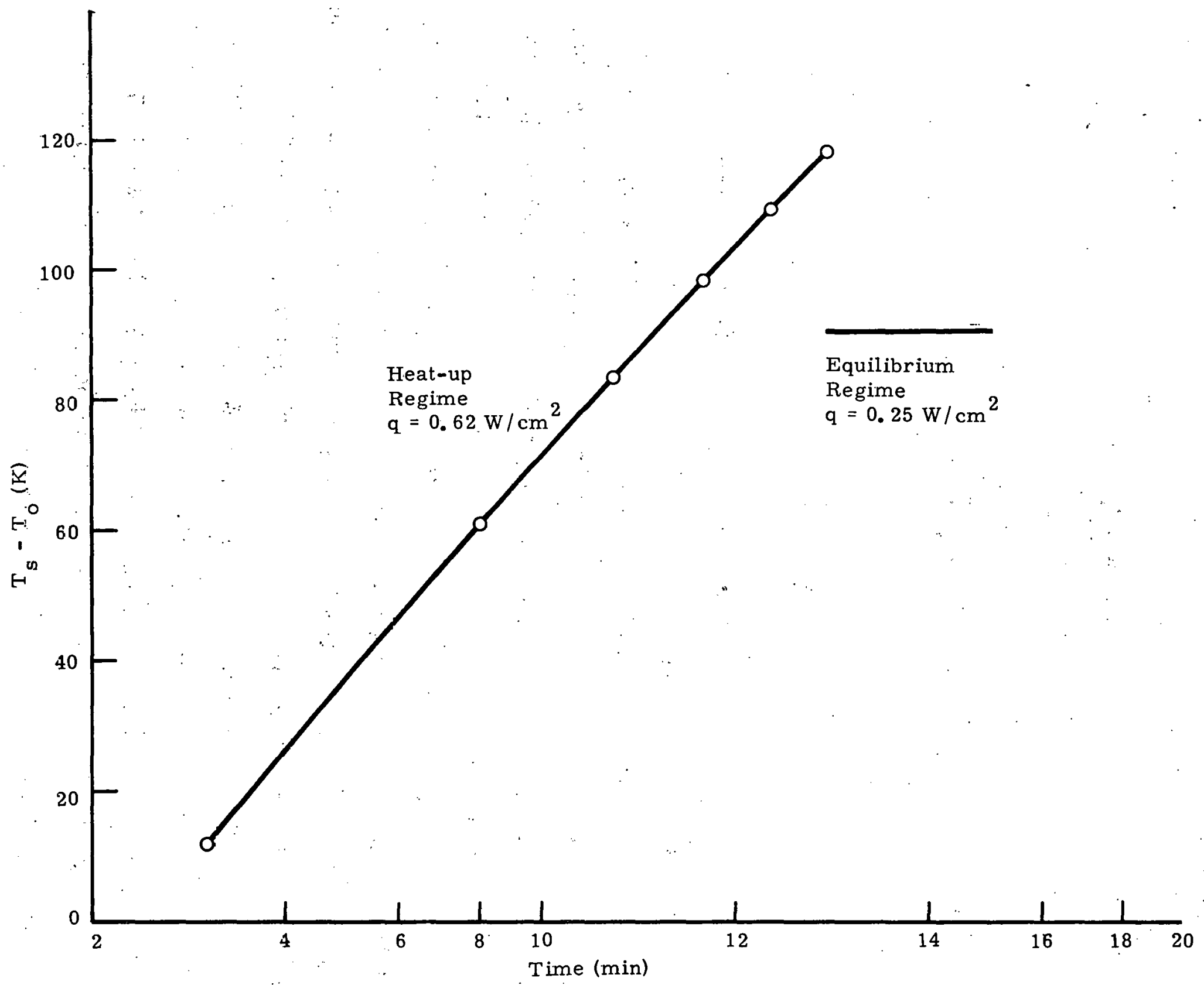

Figure 2. Temperature Response of Sphere in Sediment 
indicating that an experimental value of "thermal conductivity" could be deduced by measuring $q, I$ and $T$. This apparent thermal conductit vity, is given by,

$$
k_{a}=\frac{L}{R_{t} A_{s}}
$$

but,

$$
R_{t}^{A}=\frac{\Delta T}{q}
$$

and therefore,

$$
\mathrm{k}_{\mathrm{a}}=\frac{\mathrm{Lg}}{\Delta \mathrm{T}}
$$

For this experiment, $L$ is about $13.2 \mathrm{~cm}$ which yields a value of apparent thermal conductivity of about $0.073 \mathrm{~W} / \mathrm{cm}-\mathrm{K}$.

In the vertical direction, the temperature difference was $65.5 \mathrm{~K}$ and the distance was $20.3 \mathrm{~cm}$ between the heater surface and the surface of the sediment. The apparent thermal conductivity in the vertical direction was thus about $0.078 \mathrm{~W} / \mathrm{cm}-\mathrm{K}$. Note that the slight difference could be due to a multitude of things, e.g.; the locations of the sensors, inaccuracies in reading the thermocouples or a slight amount of natural convection. The initial portion of the temperaturetime curve appeared to be that of a heated sphere losing energy by conduction in an infinite medium. Because the heat flux was reduced during the experiment, this transient curve has no bearing upon the equilibrium temperature. In the next section of this report; a similar experiment in coarse sand will be described and there, the transient behavior will be of interest. 
At the conclusion of the experiment, the region above the sphere was carefully examined to see if the dyes had been transported upward. There was no evidence of any measurable motion in the vertical direction. although again the mud appeared to absorb most of the color of the indicator. It is clear that no appreciable channeling occurred and thus the convection was indeed low.

Finally, it should be mentioned that the "apparent thermal conductivity" determined above is only an approximation to the actual thermal conductivity of the sediments. The sample lost a large portion of its water during the duration of the test and thus behaved more like a moist clay. In spite of this, the early portion of the test during which the mud was saturated with water did not show evidence of a significant amount of natural convection. In fact, the reported value of thermal conductivity from Reference 16 for this type sediment is about $0.08 \mathrm{~W} / \mathrm{cm}-\mathrm{K}$ indicating that the situation is indeed conduction dominated.

A series of experiments were also conducted in sand to verify the analytical models of Reference 17. This section will present those cases in which the largest amount of natural convection was observed. The transient response was determined for the previously described $3.18 \mathrm{~cm}$ diameter sphere in a container of water saturated sand. The sphere was located about $25 \mathrm{~cm}$ below the surface of the sand and the container was about $30 \mathrm{~cm}$ in diameter in the horizontal direction. "Also, plotted on Figure 3 is the predicted response curve for a sphere in a purely conducting medium of infinite extent. The values of thermal conductivity and thermal diffusivity used in: the analysis were respectively $0.021 \mathrm{~W} / \mathrm{cm}-\mathrm{K}$ and $0.006 \mathrm{~cm}^{2} / \mathrm{s}$. Note that 


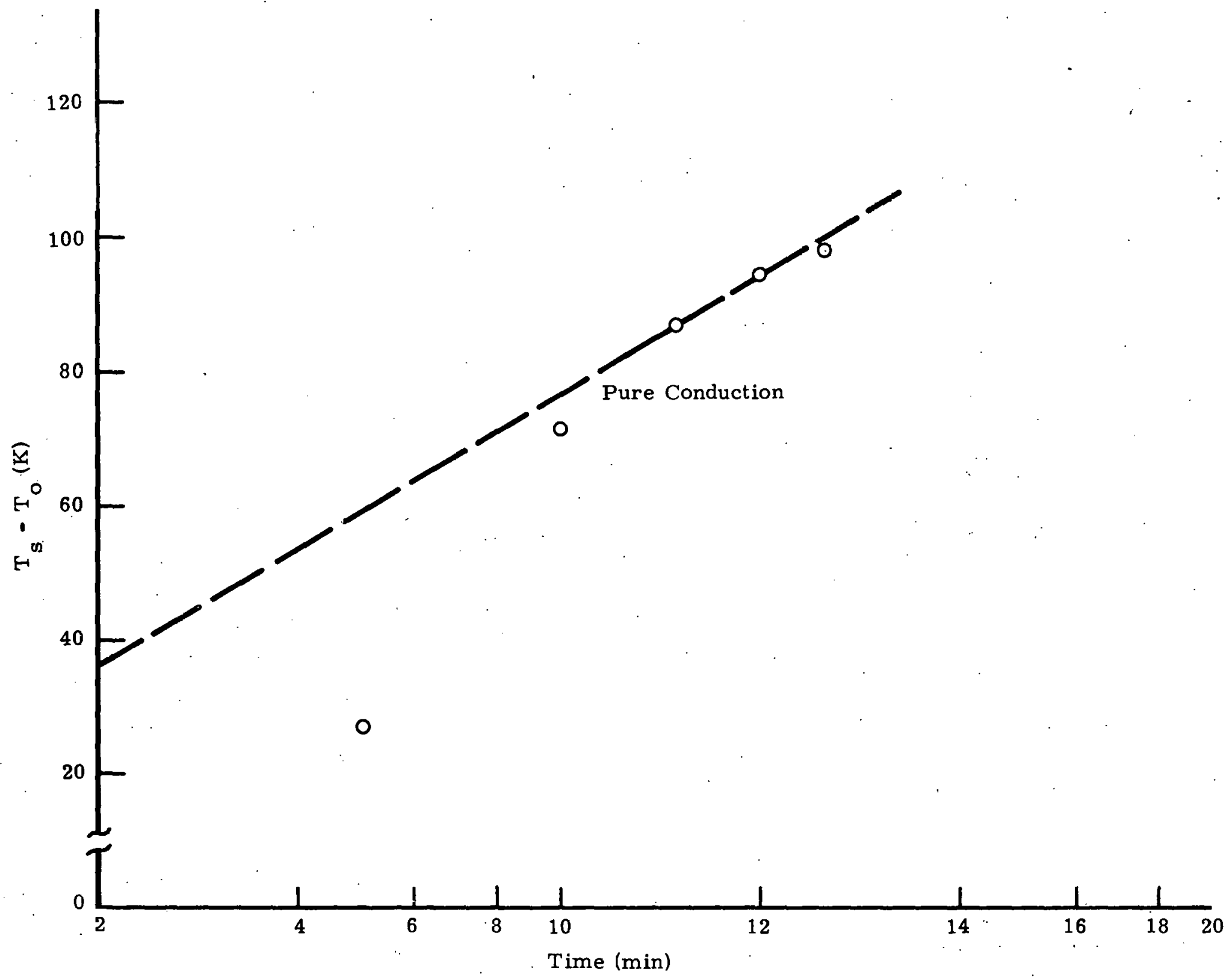

Figure 3. Temperature Response of Sphere in Coarse Sand 
except for the earliest experimental point, the agreement between predicted and observed response is excellent. The power level of the sphere was about 40 watts corresponding to a heat flux of about $1.26 \mathrm{~W} / \mathrm{cm}^{2}$.

In the next section of this report, the results of an experiment carried out in the North Pacific will be presented and analyzed. ${ }^{18}$ In this reference, the point is made that the response of the implanted heater behaved as if it were in a purely conducting medium for all times except those near the start of the experiment. Note that in the present case, the same statement could be made. Reference 18 argues that the disagreement between experiment and analysis is due to the thermal lag of the system. In fact, it should be pointed out that presenting the data in the manner of Figures 5 and 6 and Reference 18 tends to over-emphasize the early time response. The only reason for doing it here is to compare with the reference.

The record seems to indicate that the situation is pure conduction although the existence of a fairly large amount of natural convection was demonstrated using the dye-filled gelatin capsule technique. It was experimentally observed and analytically predicted by both the boundary layer and the analytical solutions that a plume velocity of some $10-46 \mathrm{~cm} / \mathrm{hr}$ exists at about 36 watts. The exact value of the velocity is unimportant. The important thing is that it was non-zero. If one asked the time required for a parcel of water to breach a $100 \mathrm{~m}$ column of this coarse sand, he would be astounded to learn that it was about one month even at these very low velocities. If nothing else, one important conclusion can be drawn from these experiments. That is: THE MERE EXISTENCE OF "CONDUCTION-LIKE" 
BEHAVIOR DOES NOT GUARANTEE THAT CONVECTION IS ABSENT. Recall that it was stated above that the value of thermal conductivity was slightIy higher than the literature value. This is analogous to the : apparent thermal conductivity discussed earlier. It is also the: argument used in attempting to correlate the data of Reference 18 . Finally, experiments conducted in sand under different heating rates and permeability show both conductive dominant and convective dominant behaviur depending on values of the experimental parameters: To conclude this section, a pilot experiment described in Reference 18 was fielded by the Geology/Genphysics group at LamonlDoherty to determine the point at which conduction heat transfer "gave way" to convection heat transfer in the sea floor sediment layer. A steel probe approximately $3.5 \mathrm{~cm}$ in diameter and $1.0 \mathrm{~m}$ long was constructed with a 300 watt internal heater. Two temperature sensors were bonded to the surface of the heater to furnish transient temperature-time data. Unfortunately, the exact location of the sensors is unknown but it was known that each is at least $\frac{3}{4} \mathrm{~m}$ from the end of the probe. Details of the experiment are presented in the reference--only the results will be summarized here.

The heater current and probe temperature for two separate runs of the device are presented in Reference 18. In the reference, the expression for an infinite line source previously mentioned is used. to correlate the data. The long time expansion is used as it would be for thermal conductivity measurements with a needle probe. 16 . $\mathrm{A}$ better approximation to the physical case is provided by the infinite length (but non-zero radius) cylinder in the finite medium, namely, 


$$
\frac{\mathrm{T}_{\mathrm{S}}-\mathrm{T}_{\mathrm{O}}}{\mathrm{Rq} / \mathrm{k}}=\left(1.128 \xi^{\frac{1}{2}}-.5 \xi+\ldots\right) .
$$

Note that $\xi=\frac{\alpha t}{\mathrm{R}^{2}}=1.0$ for times on the order of about,

$$
\frac{\mathrm{R}^{2}}{\alpha}=\frac{(1.75)^{2}}{.0022}=1392 \mathrm{~s}=23.2 \mathrm{~min} ; .
$$

(values of $\alpha$ taken from Reference 15).

For the particular values of power, surface area and thermal conductivity in the experiment, there results

$$
\begin{aligned}
& A_{S}=\pi D L=1099 \mathrm{~cm}^{2} \\
& Q=300 \mathrm{~W} \\
& q=Q / A_{S}=.273 \mathrm{~W} / \mathrm{cm}^{2} \\
& k=0.008 \mathrm{~W} / \mathrm{cm}-\mathrm{K} \\
& \alpha=0.0022 \mathrm{~cm}^{2} / \mathrm{s} .
\end{aligned}
$$

Thus, for the first two terms,

$$
T_{s}-T_{0}=1.81 t^{\frac{1}{2}}-.02 t+\ldots ; \text { in } s .
$$

This expression will correlate the data up to about $\xi=0.6$ or $t \simeq 13.9$ minutes with very little error (Figure 4 ). Beyond $\xi>2$ or $t>46.4$ minutes, the long term relation can be used with little error. For the present L/D ratio of about 28.6 , the equilibrium time is about 660 minutes beyond which the equilibrium expression holds. There will be a finite depth limit as well, but no information is given on the burial depth of the device. Presumably, the L/D effect dominates as mentioned earlier. 


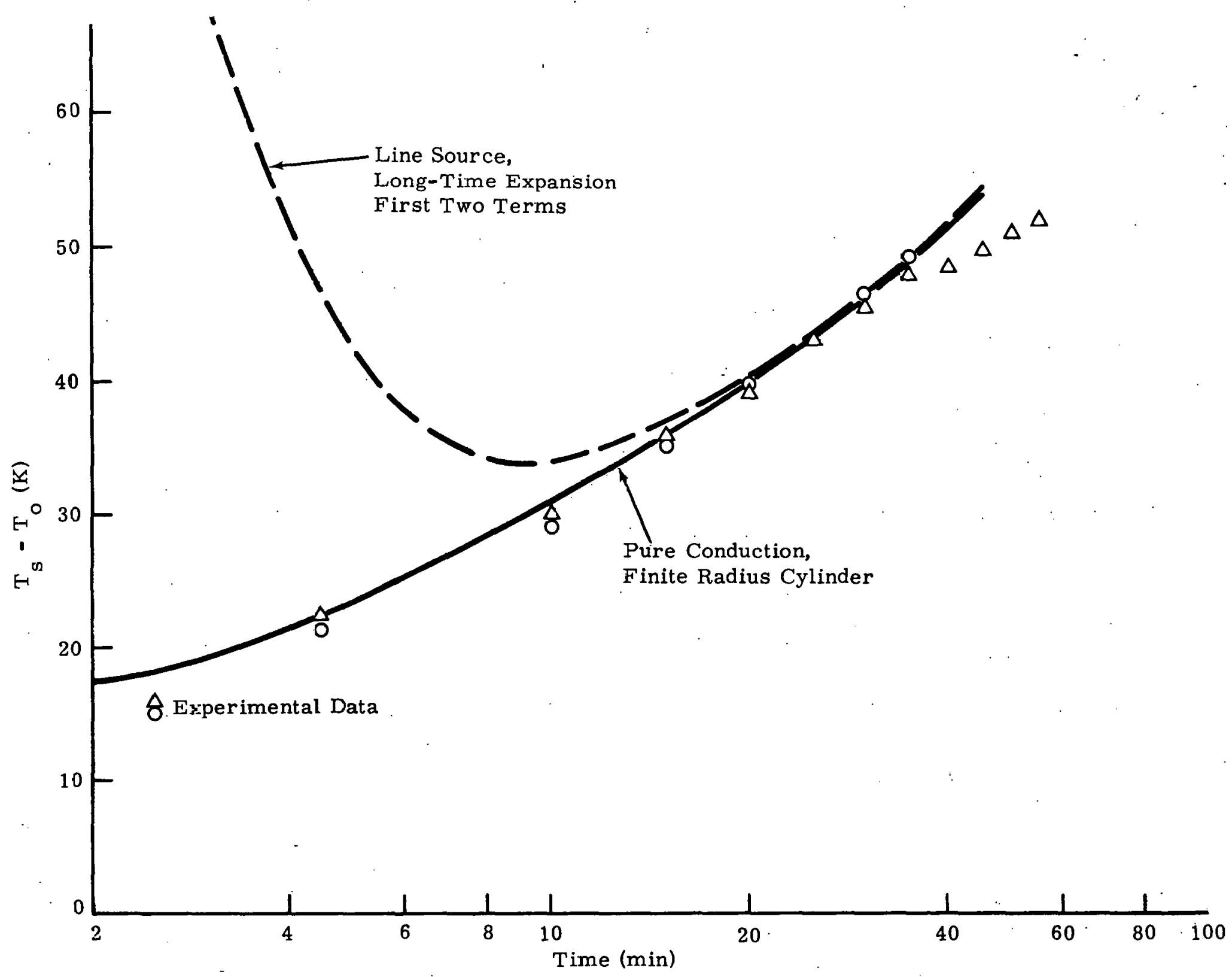

Figure 4. Temperaiure Response of Cylinder in Ocean Sediments, North-Central Pacific 
An attempt was made to reproduce the theoretical predictions of Reference 18 using the equation given as,

$$
T_{s}-T_{0}=\frac{\tilde{Q}}{4 \pi k}\left[\ln \left(\frac{4 \alpha t}{C R^{2}}\right)+\frac{R^{2}}{2 \alpha t}+\frac{(\beta-2)}{2 \beta \alpha t} R^{2} \ln \left(\frac{4 \alpha t}{C R^{2}}\right)+\ldots\right]
$$

where $\beta$ is the ratio of volumetric heat capacity of sediment to probe and the other variable notation has been changed to agree with the present paper. For $\beta^{\prime \prime} \simeq 2$ as mentioned in the reference, the last term disappears. Using the values of,

$$
\begin{aligned}
& \mathrm{k}=0.008 \mathrm{~W} / \mathrm{cm}-\mathrm{K} \\
& \alpha=0.0022 \mathrm{~cm}^{2} / \mathrm{s} \\
& \mathrm{R}=1.75 \mathrm{~cm} \\
& \mathrm{C}=1.7811=\mathrm{e}^{\gamma} .
\end{aligned}
$$

and $\tilde{Q}=300 \mathrm{~W} / 100 \mathrm{~cm}=3 \mathrm{~W} / \mathrm{cm}$, the equation becomes,

$$
T_{S}-T_{0}=29.84\left[\ln (.097 t)+\frac{11.63}{t}\right] \text {, }
$$

with $t$ in minutes.

For $t=2.5$ minutes, the value obtained from this expansion is 96.54 C. Referring to the original reference of this technique 19 or i.ts sequel, 20 Jaeger proposes the use of,

$$
T(r, t)-T_{0}=\frac{\tilde{Q}}{4 \pi k} E_{i}-\left(\frac{r^{2}}{4 \alpha t}\right),
$$

where $T(r, t)$ is the temperature at a distance $r$ from the line-source and $E_{i}$ is the exponential integral function. Note that if $r=0$ is inserted in the expression," the exponential integral of zero is 
negative infinity indicating an infinite temperature at the probe surface. It is clear that Equation (32) is hot the best one to use for short to intermediate times.

The question now to be addressed is: Given that the surface temperature of a heated object immersed in a liquid-saturated,'porous medium follows a predicted conduction response, can one conclude that convection is not present? The answer as pointed out in the section on experiments in coarse sand is "no." This does not mean that conduction solutions are worthless, however, it simply means that the temperature field is "conduction-like" but no information on the coupled velocity field can be gained from the temperature results.

\section{CONCLUSIONS}

A model has been developed for the temperature field surrounding a buried vertical circular cylinder. The cylinder is located some distance below a horizontal, isothermal surface. The only thermophysical property used in the analysis, thermal conductivity is assumed constant. Results obtained from application of the model can be used to estimate cask surface temperature as well as the temperature field surrounding the cask. This latter information can be used as input to the ion transport studies in which the various sorption coefficients are temperature dependent.

In future thermal studies, the emphasis will be on determining instability mechanisms which could provide a pathway through the sediments. Possible candidates might be fluidization of the sediment particles at the sediment water interface or a "burp" induced by 
heated buoyant fluid below the cooler surface. Unlike the present work and thermal studies which have preceeded it, this future work will be largely experimental. In fact, a series of experiments is planned to be carried out in situ. This will provide information not available from the laboratory scale tests. 


\section{REFERENCES}

1. Bishop, W. P., and Hollister, C. D., "A Program on the Ocean Basin Floors and Radioactive Materials: Data Bases for Assessing Preliminary Concepts," SLA-73-0596, Sandia Laboratories, Albuquerque, NM, September 1973.

2. B1shop, W. P., and Hollister, C. D., "Seabed Disposal--Where to Look," Nuclear Technology, Vol. 24, pp. 425-443, December'1974.

3. Hill, W. K., "A Proposal to Explore the Oceanographic option for the Dumping of High Level Radioactive Wastes," presented at the Workshop on Deep Ocean Disposal Research and Development, Washington, D.C., March 1977.

4. Webb, G. A. M., and Morley, F., "A Model for the Evaluation of the Deep Ocean Disposal of Radioactive Wastes," Rep. 14, Nat: Radiolog. Prot. Bd., 24 pp., 1973.

5. Shepherd, J. G., "A Simple Model for the Dispersion of Radioactive Wastes Dumped on the Deepsea Bed," Fish. Res. Tech., Rpt. (29), Min. of Agr., Fish., and Food, Dir. of Fish. Res., 70 pp., 1976 .

6. Schimmel, W. P., Jr., and Sullivan, W. N., "Experimental Study of Thermally Induced Buoyant Plumes in Water," SAND75-0036, Sandia Laboratories, Albuquerque, NM, September 1975.

7. Schimmel, W. P., Jr., et al., "Thermal Convection from a Localized Heat Source in Deep Ocean Sediments," SAND76-0121, Sandla Laboratories, Albuquerque, NM, April 1976.

8. Hickox, C. E., and Watts, H. A.," "steady Thermal Convection from a Concentrated Source in a Porous Medium," SAND76-0562, Sandia Laboratories, Albuquerque, NM, November 1976.

9. Sundberg, W. D., and Schimmel, W. P., Jr., "Development of Porous Media Flow with Heat Transfer Computer Code," Uncl. Sandia Memo to D. R. Anderson, dtd 11/2/76.

10. Larson, D. W., Gartling, D. K., and Hardee, H. C., Jr., "Thermal Response of Buried Radioactive Waste: Conduction Solutions," SAND75-0410, Sandia Laboratories, Albuquerque, NM, 1975. 
11. Schimmel, W. P., Jr., and Hickox, C. E., "Heat Transfer and Fluid Flow in the Deep Ocean Sediments," 1976 Annual Report-Seabed Disposal Program, SAND77-1270, Sandia Laboratories, Albuquerque, NM, 1977.

12. Carslaw, H. S., and Jaeger, J. C., Conduction of Heat in Solids, 2nd Edition, Oxford University Press, 1959.

13. Kreith, F., Principles of Heat Transfer, In-Text Educational Publishers, 1973.

14. Easton, C. R., "Conduction from a Finite-Size Moving Heat Source Applied to Radioisotope Capsule Self Burial," Thermal Conductivity, U.S. Dept. of Commerce, Spec. Publ. 302, pp. 209-218, 1968.

15. Eckert, E. R. G., and Drake, R. M., "Analysis of Heat and Mass Transfer," Fourth Edition, McGraw-Hill Book Co., NY, pp. 103-106, 1972 .

16. VonHerzen, R., and Maxwell, A. E., "The Measurement of Thermal Conductivity of Deep-Sea Sediments by a Needle-Probe Method," Journal of Geophysical Research, Vol. 61, No. 10, pp. 1557-1563, october 1959.

17. Hardee, H. C., Jr., "Boundary Layer Solutions for Natural Convection in Porous Media," SAND76-0075, Sandia Laboratories, Albuquerque, NM, January 1976.

18. Hayes, D. E., and Moody, R., "Analysis of 'Echo-Sounding' Data North-Central Pacific (Including $\mathrm{R} / \mathrm{V}$ Vema Marine Geology/ Geophysics Cruise in the North Pacific)," Progress Report, Lamont-Doherty. Geological Observatory of Columbia University, Pălisades, NY, July-October 31, 1975.

19. Jaeger, J. C., "Conduction of Heat in an. Infinite Region Bounded Internally by a Circular Cylinder of a Perfect Conductor," Australian Journal of Physics, pp. 167-179, 1956.

20:" Jaeger; J. C., "The Measurement of Thermal Conductivity and Diffusivity with Cylindrical Probes," Transactions American Geophysical Union, Vol. 39, No."4, pp. 708-710, August 1958 . 


\section{APPENDIX}

In this section, an expression will be developed for the temperature field surrounding a finite length distributed line source located vertically below an isothermal surface. This approximates the burial of a heated vertical ry inder in a semi-infinile cunducting medium (Figure $A-1$ ). As previously mentioned, this is essentially the situation which prevails in the placement of a waste cannister in the deepsea sediments. The line of reasoning taken in the analysis follows that developed in Reference 15 (for zero burial depth), except that an error in the reference will be corrected.

Consider a line of steady point heat sources located along the negative $y$ axis from $y=-H$ to $y=-H-N$ as shown in Figure $A-2$. In order to ensure that the surface $y=0$ remains at $T_{0}$, the initial temperature of the entire system, an identical row of negative steady point heat sources (heat sinks) is located from $y=H$ to $Y=H+N$. The mathematical solution of the temperature field surrounding the source can be changed to that of the sink by reflecting it about the $\ddot{y}=0$ plane and changing the sign. Because the resulting "negative" temperature field is with respect to the initial uniform temperailure, no philosophical problems should arise. Incidentally, it will be shown later that the isotherms in the lower half space are approximately ellipsoids of revolution. The analysis requires the specification of only a single medium property, thermal conductivity and it will be assumed to be constant: 


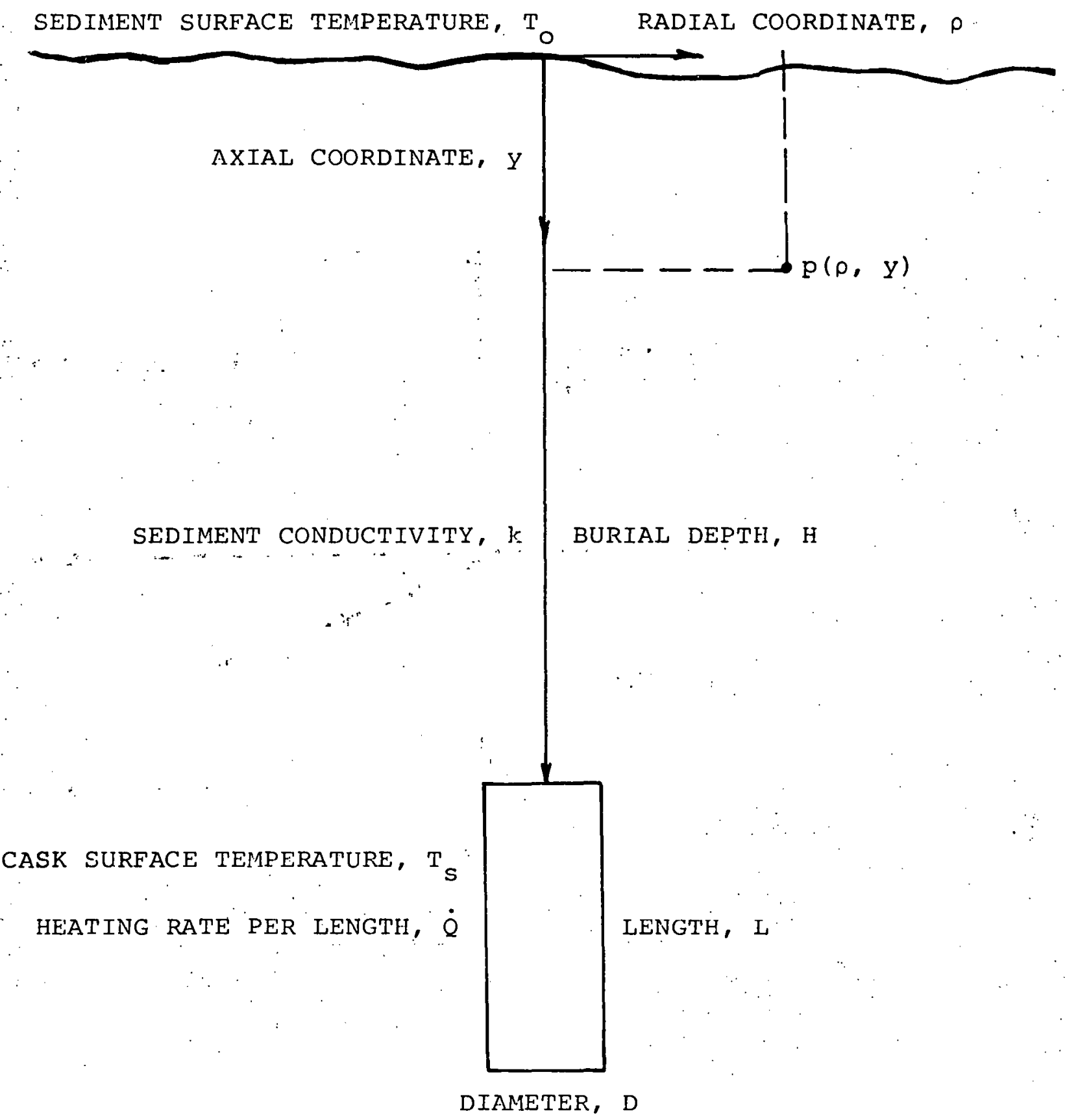

FIGURE A-1. SEABED EMPLACEMENT. 


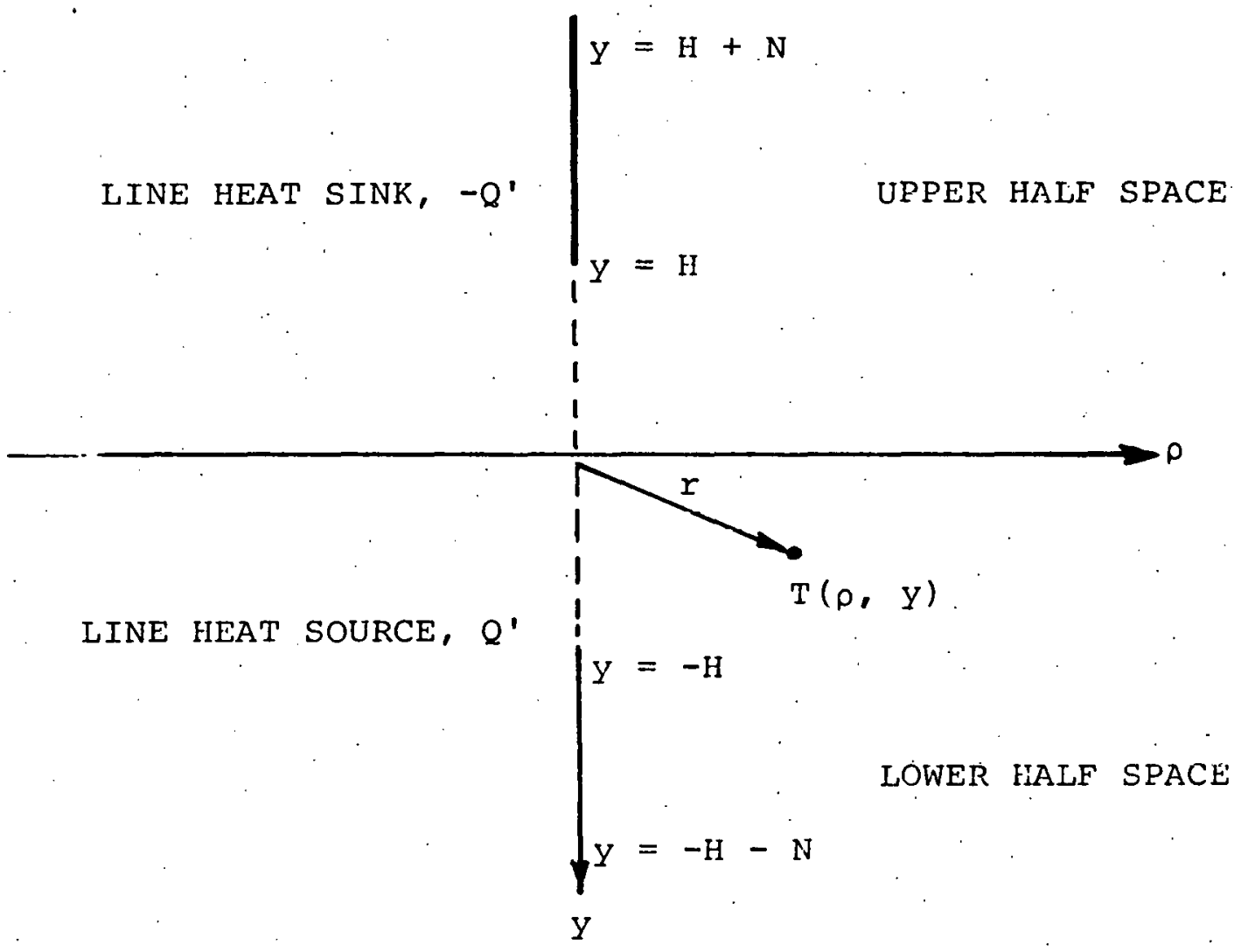

FIGURE A-2. ANALYTICAL MODEL. 
The temperature rise in a medium initially at zero temperature due to the presence of "a point heat source located at the origin is,

$$
\mathrm{T}=\frac{\mathrm{Q}}{4 \pi \mathrm{kr}},
$$

where $r=\sqrt{\rho^{2}+y^{2}}$ in units of, say $m, Q$ is the strength of the source in watts $(W)$ and $k$ is the medium thermal conductivity in $\mathrm{W} / \mathrm{m}-\mathrm{K}$.

This expression is now summed over a series of points located along the vertical axis. In the limit, the temperature increase due to the line source occurs as an integral. In terms of the coordinate system of Figure A-2, this becomes,

$$
\mathrm{T}_{1}=-\int_{-\mathrm{H}-\mathrm{N}}^{-\mathrm{H}} \frac{\mathrm{Q}^{\prime}}{4 \pi \mathrm{kr}} \mathrm{ds}
$$

where $Q^{\prime}$ is the strength per unit length and ds is an infinitesimal length of the line source. Note that the contribution of the finite line source at $r \rightarrow \infty$ is zero and thus $T_{1} \rightarrow 0$ as desired. In Reference 15, a similar argument for the case of $H=0$ was made except that the sign of the integral was taken as negative. If one integrates the integral with negative sign along an $r=r_{c}$ constant contour, it is obvious that,

$$
\begin{gathered}
\mathrm{T}_{I} \doteq-\int_{-\mathrm{H}-\mathrm{N}}^{-\mathrm{H}} \frac{Q^{\prime}}{4 \pi k r_{C}} \mathrm{ds}=-\frac{Q^{\prime}}{4 \pi k r_{C}} \int_{-H-N}^{-\mathrm{H}} \mathrm{ds} \\
\mathrm{T}_{I}=\frac{-Q^{\prime}}{4 \pi k r}[-\mathrm{H}-(\mathrm{H}-\mathrm{N})]<0
\end{gathered}
$$


and, thus, the temperature rise will be negative for all positive source strengths. This is an obvious contradiction--the sign, therefore, must be positive.

It is now stipulated that a line of heat sinks will be located along the vertical axis for $y=H$ to $y=H+N$. Arguing as before, this results in a temperature "rise" at point $r$ given by,

$$
\mathrm{T}_{2}=-\int_{\mathrm{H}}^{\mathrm{H}+\mathrm{N}} \frac{\mathrm{Q}}{4 \pi \mathrm{kr}} \mathrm{ds}
$$

This temperature rise is, of course, negative because $Q^{\prime}$ corresponds to a line sink.

Now the net temperature rise at some arbitrary $r$ will be given by the sum of the two integrals. From the figure,

$$
r^{2}=\rho^{2}+(y-s)^{2},
$$

(since we are interested only in the $y<0$ half space, the net temperature rise will be positive).

$$
\mathrm{T}=\mathrm{T}_{1}+\mathrm{T}_{2}=\int_{-\mathrm{H}-\mathrm{N}}^{-\mathrm{H}} \frac{\mathrm{Q}^{\prime}}{4 \pi \mathrm{kr}} \mathrm{ds}-\int_{\mathrm{H}}^{\mathrm{H}+\mathrm{N}} \frac{\mathrm{Q}^{\prime}}{4 \pi \mathrm{kr}} \mathrm{ds},
$$

and thus,

$$
T=\frac{Q^{\prime}}{4 \pi k r}\left\{\int_{-H-N}^{-H} \frac{d s}{\sqrt{\rho^{2}+(y-s)^{2}}}-\int_{H}^{H+N} \frac{d s}{\sqrt{\rho^{2}+(y-s)^{2}}}\right\} \text {. }
$$

substituting in the integrals

$$
\begin{aligned}
\xi & =y-s \\
d \xi & =-d s,
\end{aligned}
$$


and noting that at

$$
\begin{array}{ll}
\mathrm{s}=-\mathrm{H}-\mathrm{N}, \quad \xi=\mathrm{y}+\mathrm{H}+\mathrm{N} \\
\mathrm{s}=-\mathrm{H}, \quad \xi=\mathrm{y}+\mathrm{H} \\
\mathrm{s}=\mathrm{H}, \quad, \quad \xi=y-H \\
\mathrm{~s}=\mathrm{H}+\mathrm{N}, \quad, \quad \xi=y-H-N
\end{array}
$$

the expression becomes,

$$
T=\frac{Q^{i}}{4 \pi k}\left\{\int_{y+H+N}^{Y+H} \frac{d \xi}{\sqrt{\rho^{2}+\xi^{2}}}-\int_{y-H}^{y-H-N} \frac{d \xi}{\sqrt{\rho^{2}+\xi^{2}}}\right\} .
$$

This simplifies to,

$$
T=\frac{Q^{\prime}}{4 \pi k}\left\{\int_{Y+H}^{Y+H+N} \frac{d \xi}{\sqrt{\rho^{2}+\xi^{2}}}+\int_{y-H}^{Y-H-N} \frac{d \xi}{\sqrt{\rho^{2}+\xi^{2}}}\right\} .
$$

Carrying out the integration results in,

$$
\begin{aligned}
T= & \frac{Q^{\prime}}{4 \pi k}\left\{\ln \left[\frac{y+H+N+\sqrt{\rho^{2}+(y+H+N)^{2}}}{Y+H+\sqrt{p^{2}+(y+H)^{2}}}\right]\right. \\
& \left.+\ln \left[\frac{y-H-N+\sqrt{\rho^{2}+(y-H-N)^{2}}}{y-H+\sqrt{\rho^{2}+(y-H)^{2}}}\right]\right\}
\end{aligned}
$$

Combining the natural log functions yields, 


$$
\begin{gathered}
T=\frac{Q^{\prime}}{4 \pi k} \ln \left\{\frac{\left[y+H+N+\sqrt{\rho^{2}+(y+H+N)^{2}}\right]}{\left[y+H+\sqrt{\rho^{2}+(y+H)^{2}}\right]}\right. \\
\left.\quad \times \frac{\left[y-H-N+\sqrt{\rho^{2}+(y-H-N)^{2}}\right]}{\left[y-H+\sqrt{\rho^{2}+(y-H)^{2}}\right]}\right]
\end{gathered}
$$

Letting the burial depth, $H$, become zero results in

$T=\frac{Q^{\prime}}{4 \pi k} \ln \left\{\frac{\left[y+N+\sqrt{\rho^{2}+(y+N)^{2}}\right]\left[y-N+\sqrt{\rho^{2}+\left(y-H^{\prime}-N\right)^{2}}\right]}{\left[y+\sqrt{\rho^{2}+y^{2}}\right]^{2}}\right\}(A-11)$

In Reference 15, the expression is given as,

$$
T=\frac{Q^{\prime}}{4 \pi k} \ln \left[\frac{y+N+r_{1}}{y-N+r_{2}}\right],
$$

where,

$$
\begin{aligned}
& r_{1}^{2}=\rho^{2}+(y-N)^{2} \\
& r_{1}^{2}=\rho^{2}+(y+N)^{2} .
\end{aligned}
$$

If this incorrect expression is evaluated along $\dot{y}=0$ where $r_{1}=r_{2}$ $=\mathrm{a}$ (see Figure $A-2)$, there results,

$$
T=\frac{Q^{\prime}}{4 \pi k} \ln \left[\frac{a+N}{a-N}\right] .
$$

obviously, this is constant for various a if and only if $N=0$. Therefore, the assumption of the $y=0$ surface remaining isothermal is clearly violated and, in fact, Equation (A-12) must be wrong. 
Referring to Equation ( $A-I 1$ ) evaluated along $y=0$,

$$
T=\frac{Q^{\prime}}{4 \pi \mathrm{k}} \ln \left\{\frac{\left(\mathrm{N}+\sqrt{\rho^{2}+N^{2}}\right)\left(-N+\sqrt{\rho^{2}+N^{2}}\right)}{\rho^{2}}\right\},
$$

or,

$$
T=\frac{Q^{\prime}}{4 \pi k} \ln \left\{\frac{\rho^{2}+N^{2}-N^{2}}{\rho^{2}}\right\},
$$

which finally yields,

$$
T=\frac{Q^{\prime}}{4 \pi k} \ln (1)=0
$$

This is in line with the assumption of zero initial temperature and zero temperature along the $y=0$ plane for all values of $x$ and $z$. Returning to Equation $(A-10)$ for the general case, it is natural to inquire into the shape of the isotherms. For this exercise, it is more convenient to set Equation (A-9) equal to a constant,

$$
\begin{aligned}
T= & \text { Const }=\frac{Q^{\prime}}{4 \pi k}\left\{\ln \left[\frac{y+H+N+\sqrt{\rho^{2}+(y+H+N)^{2}}}{y+H+\sqrt{\rho^{2}+(y+H)^{2}}}\right]\right. \\
& +\ln \left[\frac{y-H-N+\sqrt{\rho^{2}+(y-H-N)^{2}}}{y-H+\sqrt{\rho^{2}+(y-H)^{2}}}\right](
\end{aligned}
$$

For finite $\mathrm{N}$ and large $\mathrm{H}$, the isotherms near the line source are déscribed approximately by,

$$
\frac{y+H+N+\sqrt{p^{2}+(y+H+N)^{2}}}{y+H+\sqrt{\rho^{2}+(y+H)^{2}}}=c \text {. }
$$


If the substitution is made for $y$,

$$
\xi=\mathrm{Y}+\mathrm{H}+\mathrm{M},
$$

where,

$$
\mathrm{m}=\frac{\mathrm{n}}{2} \text {, }
$$

then,

$$
\frac{\xi+m+r_{2}}{\xi-m+r_{1}}=c
$$

The constant, $c$, is evaluated at $\xi=0$, where $\rho=a$ constant, $b$. For $r_{1}$ and $r_{2}$, this yields,

$$
r_{1}=r_{2}=\sqrt{m^{2}+b^{2}}
$$

The constant $m$ is arbitrarily set equal to $a^{2}-b^{2}$, where $a$ is another constant, so

$$
r_{1}=r_{2}=a \text {. }
$$

Solving for $c$ in Equation $(A-20)$ results in,

$$
c=\frac{a+m}{a-m} \text {. }
$$

For the general case, Equation $(A-20)$ specifies,

$$
\left(\xi+m+r_{2}\right)(a-m)=\left(\xi-m+r_{1}\right)(a+m) \text {, }
$$

which simplifies to,

$$
\frac{\rho^{2}}{a^{2}-m^{2}}+\frac{\xi^{2}}{a^{2}}=1
$$


but $a^{2}-m^{2}=b^{2}$; so finaliy,

$$
\frac{\rho^{2}}{b^{2}}+\frac{\xi^{2}}{a^{2}}=1
$$

This is the equation of an ellipse of major axis $2 a$ and minor axis $2 \mathrm{~b}$ centered at the origin. In terms of physical coordinates,

$$
\frac{\rho^{2}}{b^{2}}+\frac{(y+H+N / 2)^{2}}{a^{2}}=1 \text {. }
$$

which is the same size ellipse centered about the $\rho=0, \dot{y}=-\mathrm{H}-\mathrm{N} / 2$ point. The same procedure applied to the second part of Equation. $(A-17)$ indicates that the negative isotherms about the line sink are ellipses centered about $\mathrm{y}=\mathrm{H}+\mathrm{N} / 2$. The resulting isotherms due to the presence of both the source and sink can, in general, be obtained from,

$$
\begin{aligned}
& \left\{\left[\begin{array}{l}
y+H+N+\sqrt{\rho^{2}+(y+H+N)^{2}} \\
y+H+\sqrt{\rho^{2}+(y+H)^{2}}
\end{array}\right]\right. \\
& \left.\quad x\left[\frac{y-H-N+\sqrt{\rho^{2}+(y-H-N)^{2}}}{y-H+\sqrt{\rho^{2}+(y-H)^{2}}}\right]\right\}=c \cdot(A-28)
\end{aligned}
$$

Selected isotherms are plotted in Figure $A-3$ for the case $H / N=2$. 
If one of these isotherms is selected as the physical object, the conductive field can be solved explicitly. Rather than making an arbitrary choice, the surface areas of the ellipsoid and the physical cylinder will be matched. Before doing this, the temperature rise of the ellipsoid will be determined.

since the temperature of the ellipsoid surface is constant, it can be evaluated at any value of $(\rho, y)$ consistent with the ellipse equation. Equation $(A-9)$ is thus evaluated at $\rho^{-}=b, y=-H-m$. As before, $m=\mathrm{N} / 2$.

$T=\frac{Q^{\prime}}{4 \pi k}\left\{\ln \left[\frac{m+\sqrt{b^{2}+m^{2}}}{-m+\sqrt{b^{2}+m^{2}}}\right]+\ln \left[\frac{-2 H-3 m+\sqrt{b^{2}+(2 H+3 m)^{2}}}{\sqrt{b^{2}+(2 H+m)^{2}}}\right]\right\} \cdot(A-30)$

Rewriting this slightly,

$\frac{T}{Q^{\prime} / 2 \pi k}=\frac{1}{2}\left\{\ln \left[\frac{a+m}{a-m}\right]+\ln \left[\frac{-2 H-3 m+\sqrt{b^{2}+(2 H+3 m)^{2}}}{-2 H-m+\sqrt{b^{2}+(2 H+m)^{2}}}\right]\right\}$

The first term can be rewritten,

$$
\ln \left[\frac{a+m}{a-m}\right]=\ln \sqrt{\frac{a+m}{a-m}}
$$

Multiplying the numerator and denominator of the log function argument of $\sqrt{a+m}$ results in,

$$
\ln \sqrt{\frac{(a+m)^{2}}{a^{2}-m^{2}}}=\ln \left(\frac{a+m}{b}\right)
$$

which becomes,

$$
\ln \left(\ell+\sqrt{\ell^{2}-1}\right)
$$


The final temperature expression is,

$$
\frac{T}{Q^{1} / 2 \pi k}=\ln \left(\ell+\sqrt{\left.\ell^{2}-1\right)}+\frac{1}{2} \ln \left(\frac{2 h+3 n-r_{3}}{2 h+n-r_{4}}\right)\right. \text {, }
$$

where,

$$
\begin{aligned}
& \mathrm{h}=\mathrm{H} / \mathrm{b} . \\
& \mathrm{n}=\mathrm{m} / \mathrm{b}=\sqrt{\ell^{2}-1} \\
& r_{3}=\sqrt{1+(2 h+3 n)^{2}} \\
& r_{4}=\sqrt{1+(2 h+n)^{2}} .
\end{aligned}
$$

All the depth of burial dependence is in the second term so that as $h$ tends to infinity only the first term remains. This agrees with Reference 14 (except for the negative sign) which considers' a finite length cylinder in an infinite medium. All that remains is to determine the relationship between the isothermal ellipsoid and the actual buried cylinder.

Recall that the surface area of a right circular cylinder is given by,

$$
A_{C}=\pi D\left(L+\frac{D}{2}\right)
$$

where $\mathrm{L}$ and $\mathrm{D}$ are the length and diameter, respectively. For a prolatc ophcroid (ellipsuid of revolution about the major axis), the surface area can be calculated from,

$$
A_{e}=2 \pi b\left(b+\frac{a}{\varepsilon} \sin ^{-1} \varepsilon\right) \text {, }
$$

where $2 a$ is the major axis, $2 b$ is the minor axis and, 


$$
\varepsilon=\sqrt{1-\frac{b^{2}}{a^{2}}}<1.0
$$

Equating the two results in,

$$
2 b^{2}+\frac{2 a b}{\varepsilon} \sin ^{-1} \varepsilon=L D+\frac{D^{2}}{2} .
$$

The following substitutions are made,

$$
\begin{aligned}
& \ell=L / D \\
& \beta=b / d \\
& \alpha=a / D
\end{aligned}
$$

resulting in,

$$
2 \beta^{2}+2 \alpha \beta\left(\frac{\sin ^{-1} \varepsilon}{\varepsilon}\right)=\ell+\frac{1}{2},
$$

where now,

$$
\varepsilon=\frac{1}{l} \sqrt{\ell^{2}-1}
$$

In addition to matching the surface areas, it is desirable for the two objects to keep the same proportion so it is specified that,

$$
\frac{\alpha}{\beta}=\frac{a}{b}=\ell
$$

Equation $(A-35)$ can thus be solved for $B$,

$$
\beta=\left\{\frac{l+\frac{3}{2}}{\frac{2+2 \ell \sin ^{-1} \varepsilon}{\varepsilon}}\right\}^{\frac{l}{2}},
$$


and $\alpha$ is given by

$$
\therefore \quad, \quad \alpha=\ell \beta=
$$

In terms of physical dimensions of the ellipsoid,

$$
a=L\left\{\frac{\ell+\frac{1}{2}}{\frac{2+2 \ell \sin ^{-1} \varepsilon}{\varepsilon}}\right\}^{\frac{1}{2}},
$$

and,

$$
\mathrm{b}=\mathrm{D}\left\{\frac{\ell+\frac{1}{2}}{\frac{2+2 \ell \sin ^{-1} \varepsilon}{\varepsilon}}\right\}^{\frac{1}{2}} .
$$

Note that the quantity in brackets is only a function of $\ell$ and is tabulated below:

$\begin{array}{ll}\ell & \left.\left\{\frac{\ell+\frac{1}{2}}{\varepsilon}\right\}^{\frac{2+2 l \sin ^{-1} \varepsilon}{\varepsilon}}\right\}^{\frac{2}{2}} \\ 2 & .61 \\ 3 & .60 \\ 4 & .59 \\ 5 & .59 \\ 10 & .58 \\ 20 & .57 \\ 50 & .57 \\ 100 & .57\end{array}$


Using a value of $1.2 \mathrm{~L}$ for the major axis and $1.2 \mathrm{D}$ for the minor axis, lengths of the ellipsoid should be sufficiently accurate for this model. This, then, is the correspondence between the model and the actual physical situation. Limits of applicability of the model are unknown at present, however, so care should be taken to avoid high heat flux to length values. 
DISTRIBUTION :

Woods Hole Oceanographic Institute

(2)

Woods Hole, MA 02545

Attn: V. T. Bowen

C. D. Hollister

D. A. Deese

Harvard University

9 Divinity Avenue

Cambridge, MA

02138

T. E. Ewart (2)

Program Manager, Ocean Physics Group

Applied Physics Laboratory

University of Washington

1013 Northeast 40 th Street

Seattle, WA

Attn: T. E. Ewart

L. R. Olson

U.S. Department of Energy

Washington, D.C. 20545

Attn: M. S. Kearney, DECT

D. H. Hamilton, Jr., Division of Biomedical and

Environmental Research

D. E. Hayes

Lamont-Doherty Geologic Observatory

Palisades, NY 10964

School of Oceanography (2)

University of. Rhode Island

Kingston, RI 03881

Attin: G. R. Heath

A. J. Silva

Scripps Institute of Oceanography

(4)

$$
\begin{aligned}
& \text { LaJolia, } \text { GA } 92037 \\
& \text { Attn: } \text { R. R. Hessler } \\
& \text { J. A. McGowan } \\
& \text { K. Smith } \\
& \text { A. A. Yayanos }
\end{aligned}
$$

K. Keil

Geology Department

University of New Mexico

Albuquerque, NM 87131 
DISTRIBUTION (cont):

1000 G. A. Fowler

1200 L. D. Smith

1260 K. J. Touryan

1261 D. F. McVey

1261 W. P. Schimmel, Jr. (25)

1262 H. C. Hardee

1262 C. E. Hickox

1280 . T. B. Lane

$2513^{\circ}$ A. B. Donaldson

5000 A. Narath

5200 E. H. Beckner

5300 O. E. Jones

5330 R. W. Lynch

5336 D. R. Anderson

5336 D. M. Talbert

5400 A. W. Snyder

5410 D. J. McCloskey

5430 R. M. Jefferson

5700 J. H. Scott

5710 G. E. Brandvold

5730 H. M. Stoller

5740 V. L. Dugan

5832 R. W. Rhode

8000 T. B. Cook, Jr.

8110 D. Hartley

8120 W. E. Alzheimer

8121 A. F. Baker

8266 E. A. Aas (2)

3141 C. A. Pepmueller (Actg)

3151 W. L. Garner (3)

for ERDA/TIC (Unlimited Release) 